\title{
Quantum Confinement in InAs/GaAs Systems with Self-Assembled Quantum Dots Grown Using In-Flush Technique
}

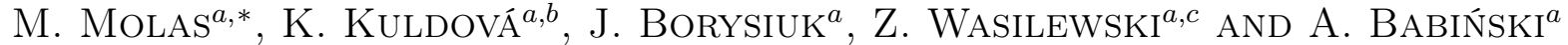 \\ ${ }^{a}$ Institute of Experimental Physics, Faculty of Physics, University of Warsaw, Hoża 69, 00-681 Warszawa, Poland \\ ${ }^{b}$ Institute of Physics of the AS CR, Cukrovarnická 10/112, CZ-182 21 Praha 8, Czech Republic \\ ${ }^{c}$ Institute for Microstructural Sciences, National Research Council Ottawa, Ontario, K1A 0R6, Canada \\ The effect of In-flush technique application to the MBE-grown structure with self-assembled quantum dots \\ is investigated in this work. The microphotoluminescence from structures with the InAs/GaAs dots grown \\ with and without the In-flush has been investigated. We focus our attention on "not fully developed" dots, \\ which can be clearly distinguished in the spectrum. The dots have also been identified in the transmission \\ electron microscopy analysis of the structures. The In-flush does not influence a broad energy range of those \\ features. Instead we have found that the anisotropic exchange energy splitting of neutral excitons confined \\ in those in the structure grown with In-flush is substantially lower that the splitting in the structure with no \\ In-flush. This observation confirms that the In-flush leads not only to better uniformity of self-assembled quantum \\ dots but also to reduction of lateral potential, anisotropy, which is believed to result in the neutral exciton splitting.
}

PACS: 78.55.Cr, 78.67.Hc

\section{Introduction}

Self-assembled InAs quantum dots (SAQDs) grown on a GaAs substrate are intensively studied for numerous applications and interesting fundamental physics. It is well known that growth conditions critically affect their morphology. In particular, the application of In-flush technique [1], which leads to formation of flat QDs, results in narrowing and the blue shift of inhomogeneous energy distribution of dots and characteristic excitonic shell structure $[2,3]$. Moreover formation of In-composition fluctuations in a wetting layer (WL) was observed in such structures [4]. In this communication we study the effect of the technique on the quantum confinement in the structures with InAs/GaAs SAQDs. We show that the In-flush technique affects the asymmetry exchange splitting of neutral excitons in "not-fully developed" quantum dots.

\section{Experimental procedure}

The samples investigated in this work was grown by using molecular beam epitaxy and indium-flush technique [1]. It contains a $200 \mathrm{~nm}$ GaAs buffer on a semi-insulating GaAs substrate. Following the superlattice sequence was $20 \mathrm{~nm}$ thick AlAs, followed by a $20 \mathrm{~nm}$

\footnotetext{
* corresponding author; e-mail: maciej.molas@gmail.com
}

GaAs. This sequence repeated 20 times. Then GaAs layer was formed by supplying $20 \mathrm{~nm}$ of GaAs at $510^{\circ} \mathrm{C}$ on a $600 \mathrm{~nm}$ thick GaAs buffer. After this a growth was started of $5.6 \AA$ InAs QD with gradient of "nominal" thickness of InAs, which capped with a $20 \mathrm{~nm}$ GaAs cap for sample V0718 (without indium-flush) or with a $5 \mathrm{~nm}$ GaAs cap for V0719 (with indium-flush technique). The structure was completed with $85 \mathrm{~nm} \mathrm{GaAs}$ for V0718 or $100 \mathrm{~nm}$ GaAs for V0719. A set of mesa structures was prepared on the sample in order to reproducibly locate a dot, optically addressed. The Nd:YAG laser $(\lambda=532 \mathrm{~nm})$ was used for photoluminescence (PL) excitation. The PL was measured at $T=4.2 \mathrm{~K}$. The excitation light was delivered and the PL was collected via a microscope objective (spot size a few $\mu \mathrm{m}$ ), dispersed by using a $1 \mathrm{~m}$ monochromator and detected by a CCD camera. The polarization of emitted light was analyzed by using a linear polarizer placed before the spectrometer.

\section{Result and discussion}

The PL spectrum from the investigated structure consists of the emission from the GaAs barrier and self-assembled QD [5], as well as the emission related to the WL. The typical PL spectrum dependence on position on studied samples measured on a ten-micron-size mesa $(10 \mu \mathrm{m} \times 10 \mu \mathrm{m})$ observed in the energy range $1.24-1.48 \mathrm{eV}$ (see Figs. 1 and 2).

We have analyzed the dependence of the micro-PL intensity of spectra on position on investigated samples. 


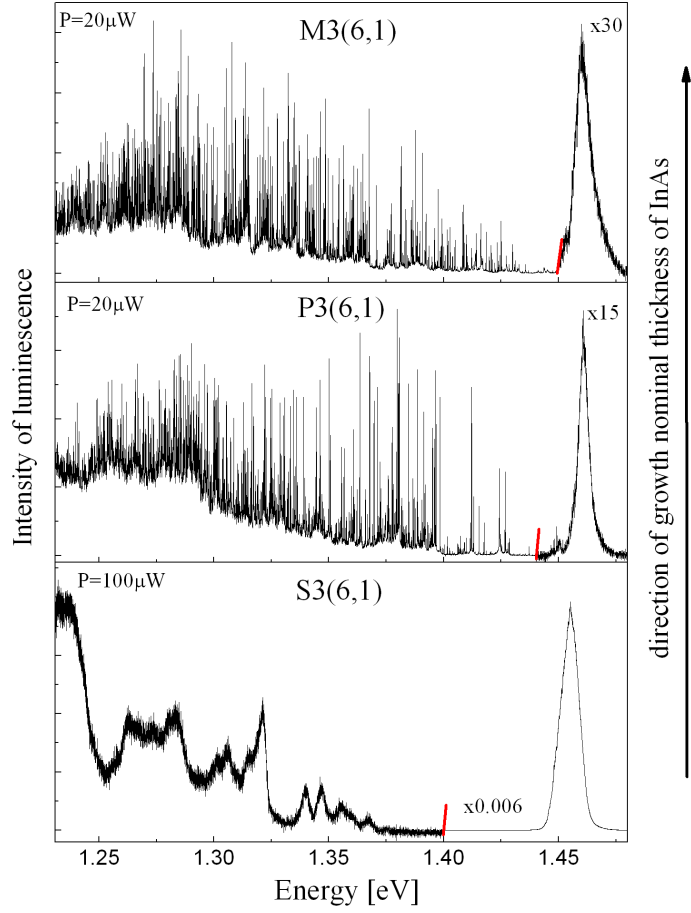

Fig. 1. Micro-PL spectra of the V0718 sample (no In-flush) taken from 3 mesas $\left(10 \times 10 \mu \mathrm{m}^{2}\right)$.

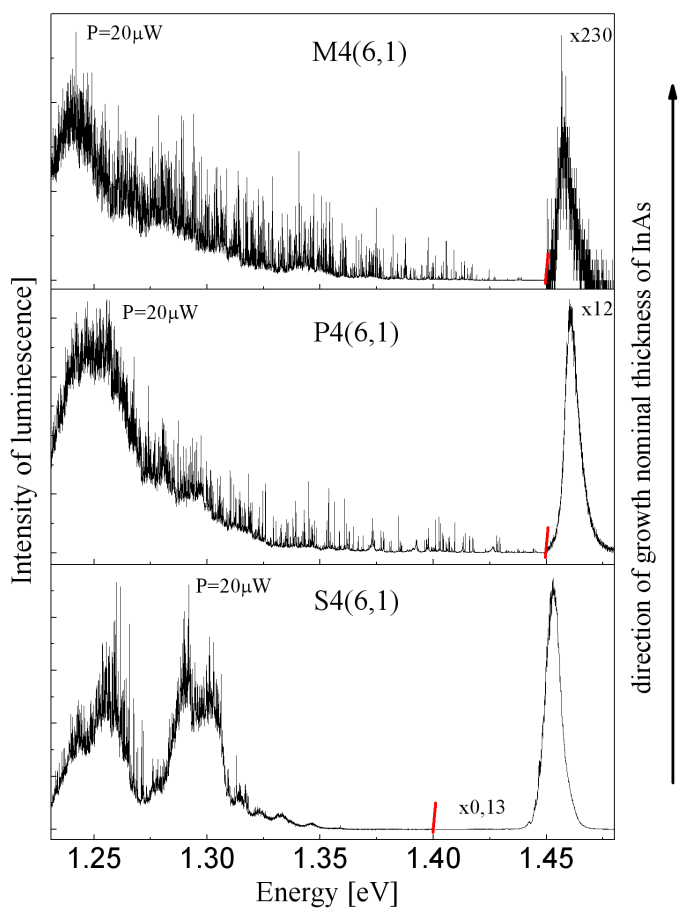

Fig. 2. Micro-PL spectra of the V0719 sample (with In-flush) taken from 3 mesas $\left(10 \times 10 \mu \mathrm{m}^{2}\right)$.
We observed an increase of the SAQDs with increasing of "nominal" deposition thickness of InAs. The emission from the QDs distribution was observed to shift from $1.07 \mathrm{eV}$ for V0718 to $1.16 \mathrm{eV}$ for V0719 (at room temperature) as a result of In-flush application. Using a two-oscillator model [6] the energy of the ground state of SAQDs at liquid $\mathrm{He}$ (LHe) temperature was estimated to shift from $1.15 \mathrm{eV}$ to $1.24 \mathrm{eV}$. The relative intensity of the SQDs-emission and the WL-emission reflects the density of SQDs. The emission from NQDs was observed only in a few positions, which suggests a local character of In composition fluctuations in the WL.

The low-temperature spectrum in samples with higher densities of SAQDs comprised also a series of sharp lines in the energy range of $1.30-1.45 \mathrm{eV}$.

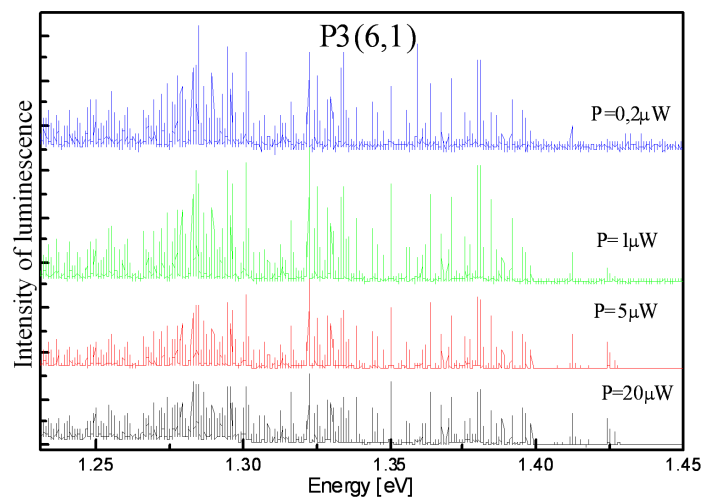

Fig. 3. Micro-PL spectra of the V0718 sample (without In-flush) taken from $10 \times 10 \mu \mathrm{m}^{2}$ mesa. Spectra normalized by power of laser.

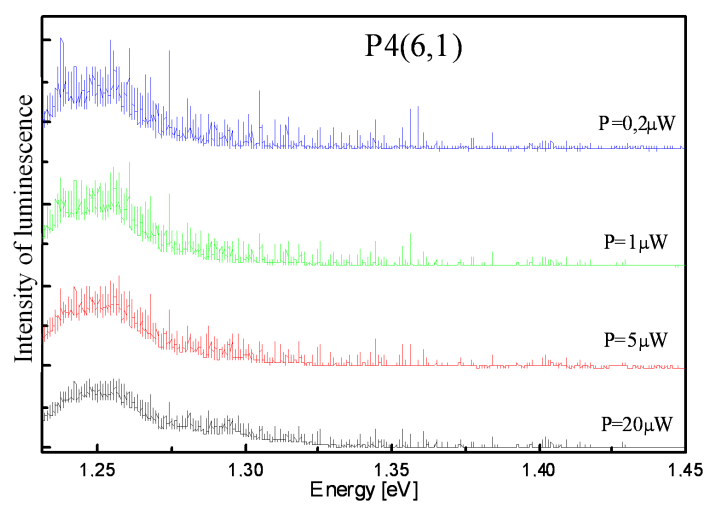

Fig. 4. Micro-PL spectra of the V0719 sample (Inflush) taken from $10 \times 10 \mu \mathrm{m}^{2}$ mesa. Spectra normalized by power of laser.

The power dependence of the spectra from both samples are shown in Figs. 3 and 4 . It can be seen that the sharp lines do not disappear with decreasing laser power. This suggests that those lines are related to smaller quantum dots than the SAQDs, which we refer to as "not fully developed" quantum dots (XQDs). Density of XQDs is 


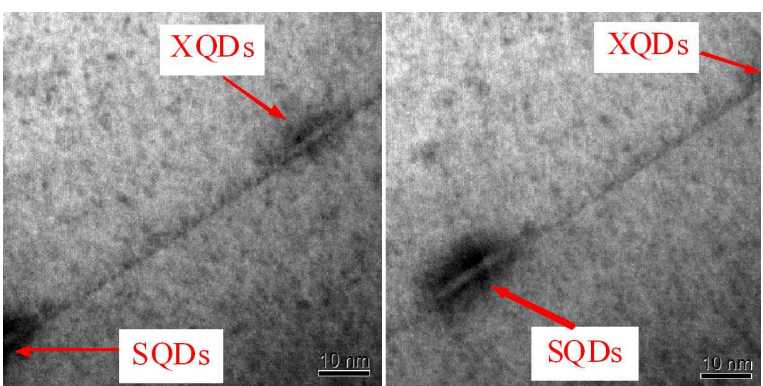

Fig. 5. The TEM image of region with XQDS from sample V0719.

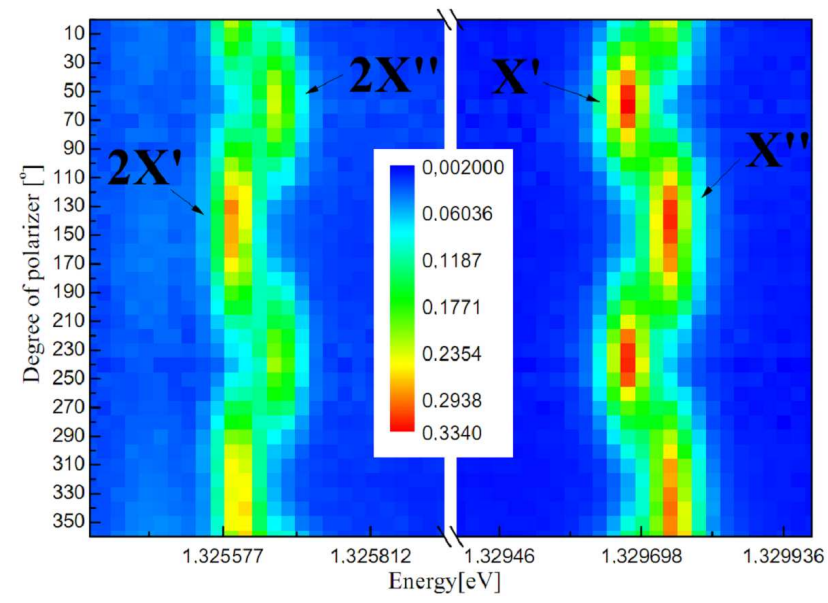

Fig. 6. Normalized polarization-sensitive micro-PL spectra of the excitons localized in a single XQDs from V0718.

much lower than density of SAQDs. No effect of In-flush on the sharp lines energy distribution suggests its attribution to XQDs with heights lower than $5 \mathrm{~nm}$ (height of the first layer cap on V0719). We relate those lines to "not-fully developed" quantum dots, which are smaller than the dots from main QDs size distribution.

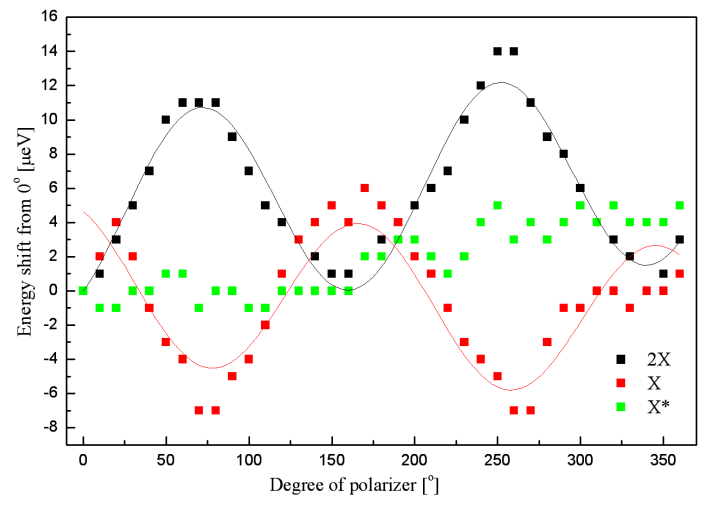

Fig. 7. Energy shift dependence on degree of polarizer of the excitons localized in a single XQDs from V0719.
To confirm the presence of XQDs in the investigated samples, the transmission electron microscopy (TEM) analysis was performed. Two quantum dots of a different size have been observed in the image of the V0719 sample (Fig. 5).

In order to investigate single sharp lines, we have performed an analysis of the emission lines from the "not-fully developed dots" (see Figs. 6 and 7) from $2 \mu \mathrm{m} \times$ $2 \mu \mathrm{m}$ mesas.

We have performed an analysis of the emission lines from the (see Figs. 6 and 7). We identified neutral and charged excitons confined in the dots. The optical anisotropy of the neutral excitons due to anisotropic exchange interaction confined in the dots was found to be different for both samples. The splitting observed for the sample with no In-flush (of the order of $70 \mu \mathrm{eV}$ ) was usually a few times larger than anisotropy in the sample with the In-flush (of the order of $10 \mu \mathrm{eV}$ ).

Our observation suggests that the application of In-flush decreases not only the dot's energy distribution but also the symmetry of lateral localization potential. Although our analysis concerns the "not-fully developed" quantum dots we believe that our observation is of more general character and the application of In-flush technique affects also the symmetry of SAQDs, which may potentially be of importance for applications of those dots for quantum information processing.

\section{References}

[1] Z.R. Wasilewski, S. Fafard, J.P. McCaffrey, J. Cryst. Growth 201, 1131 (1999).

[2] M. Korkusiński, M. Zieliński, P. Hawrylak, J. Appl. Phys. 105, 122406 (2009).

[3] A. Babiński, M. Potemski, S. Raymond, J. Lapointe, Z. Wasilewski, Phys. Rev. B 74, 155301 (2006).

[4] A. Babiński, J. Borysiuk, S. Kret, M. Czyz, A. Golnik, S. Raymond, Z.R. Wasilewski, Appl. Phys. Lett. 92, 171104 (2008).

[5] For more details on the QDs emission see A. Babinski, M. Potemski, S. Raymond, J. Lapointe, Z. Wasilewski, Phys. Rev. B 74, 155301 (2006).

[6] G. Ortner, M. Schwab, M. Bayer, R. Pässler, S. Fafard, Z. Wasilewski, P. Hawrylak, A. Forchel, Phys. Rev. B 72, 085328 (2005). 